\title{
The Efficacy and Safety of a Patent Pending Combination of Ginger and Goldenrod Extracts on the Management of Cold Symptoms: A Randomized, Double-Blind Controlled Trial
}

\author{
Johane Guay $^{\mathbf{1}}$, Pierre Champagne ${ }^{2}$, Pascal Guibord ${ }^{3}$, Joerg Gruenwald ${ }^{\mathbf{4}}$ \\ ${ }^{1}$ Biopharmacopae Inc., Quebec City, Canada; ${ }^{2}$ Institut Privé de Chirurgie, Quebec City, Canada; ${ }^{3}$ Statistician, Lachine, Canada; \\ ${ }^{4}$ analyze \& realize ag, Berlin, Germany. \\ Email: hstier@analyze-realize.com
}

Received September 26 ${ }^{\text {th }}$, 2012; revised October $23^{\text {rd }}, 2012$; accepted November $1^{\text {st }}, 2012$

\begin{abstract}
Objective: In a randomized, double-blind, exploratory, active-controlled trial, the efficacy and safety of a patentpending combination of Ginger and Goldenrod extracts (BDI-630) in alleviating cold symptoms in community-dwelling adults was compared to a combination of standardized amounts of Echinacea (EC) components. Methods: 44 healthy adults, experiencing new onset of cold symptoms were randomly assigned to receive either BDI-630 (900 mg) or EC (500 mg) twice daily for 10 days. The severity of cold symptoms and the quality of life was assessed by self-reporting of subjects using the Wisconsin Upper Respiratory Symptom Survey (WURSS-21@2004). Results: The intent-to-treat population (ITT) consisted of 44 subjects ( $\mathrm{n}=22$ for BDI-630; $\mathrm{n}=22$ for EC). The modified ITT (mITT) population consisted of 40 subjects, excluding four subjects with major protocol deviations related to inclusion/exclusion criteria and/or use of prohibited drugs from the efficacy analysis ( $n=3$ for BDI-630 and $n=1$ for EC). Results indicated a significant difference between the two groups: the superiority of BDI-630 over EC was particularly noticeable between Day 1 and Day 7, as demonstrated by a 14-fold difference of the mean percentage of change of total score from baseline in the mITT population. Adverse events (AEs) following the intake of BDI-630 were mostly limited to mild gastrointestinal intolerance in less than $10 \%$ of the subjects. Conclusions: BDI-630 was shown to be more effective than EC in alleviating cold symptoms in the adult population, particularly during the first 7 days of treatment. BDI-630 was very well tolerated by all subjects.
\end{abstract}

Keywords: Randomized Double-Blind Active-Controlled Trial; Common Cold

\section{Introduction}

The common cold is a very frequent illness in industrialized societies; it is the leading cause of doctor visits in the United States and Canada, and one of the most frequent causes of absenteeism at work. In fact, the common cold generates an enormous economic burden including lost productivity and treatment costs [1]. The National Center for Health Statistics estimates that 21\% (62 million) Americans are affected with symptoms of the common cold and flu each year [2]. These incidence rates are similar across industrialized countries and underscore the impact of the cold and flu season on commerce and health care. In the US this represents $\$ 17$ billion per year in direct health care costs and approximately $\$ 22.5$ billion per year in indirect costs (e.g., lost wages and productivity) [1].
The common cold is caused by a variety of viruses, most of the time, the rhinovirus and corona types $[3,4]$. There is currently no treatment for the common cold; therefore, therapies are focused on symptom relief. Although OTC drugs are widely used, they have important side effects and contraindications. Clinical studies using the scientific method supporting cough and cold herbal preparations prove inexistent or elusive. In particular, Echinacea's clinical efficacy demonstrations against colds were found contradictory and non-conclusive, but the herb still remains popular among consumers around the world.

Ginger (Zingiber officinale Roscoe) has been used for thousands of years as a treatment for a wide variety of ailments, but specifically for nausea, motion sickness and diarrhea [5]. The active ingredients in subject come from its volatile oil, sesquiterpenes with zingiberene as the 
main constituent [5]. Goldenrod (Solidago Virgaurea) consists of woody stems and yellow flowers that are found in open areas along roads or open meadows. Goldenrod has been traditionally used in the treatment of inflammation, wounds, and minor urinary tract complaints. The main chemical compounds are a wide range of active ingredients, such as flavonoids, saponins, hydroxycinnamates and mineral elements, which are responsible for its characteristic anti-inflammatory, spasmolytic and diuretic properties [6].

In vitro and pre-clinical studies performed on the patent-pending combination of Ginger and Goldenrod extracts (BDI-630) have demonstrated the effectiveness of the combination to inhibit the growth of Rhinoviruses and the Influenza virus (data unpublished). Moreover, the effectiveness of BDI-630 tested against oseltamivir and a leading Canadian natural health brand against colds, has been proven to be comparable to oseltamivir, and significantly superior to the leading natural health brand, in extending life span of mice inoculated intranasally with a lethal dose of the Influenza virus (unpublished data). Based on these results and on the fact that the herbal extracts within BDI-630 are individually recognized safe by Canadian and European Pharmacopeia, and documented in the German Commission E monographs, BDI-630 was deemed pertinent for clinical testing as a new safe natural option against colds.

The present exploratory study investigated the effects of BDI-630 on the severity of cold symptoms and quality of life in individuals suffering from cold symptoms in Eastern Canada. The patent-pending combination of plant extracts in BDI-630 was compared against a natural health product containing a combination of standardized amounts of Echinacea (EC) components over 10 days of treatment.

\section{Materials and Methods}

The study protocol, amendments, the Informed Consent Form (ICF), subject questionnaire/diary and recruitment ads were approved by an external ethic committee. The study was conducted in compliance with ethical principles that have their origin in the Declarations of Helsinki and its amendments and the International Conference on Harmonization (ICH) Guideline for Good Clinical Practices (GCP) (E6). Applicable national laws and any other relevant laws (e.g., privacy and consent legislation) were followed.

\subsection{Design}

This study was designed as a randomized, double-blind design. Neither the Investigator nor the subject knew the treatment group to which the subject was assigned.

\subsection{Subjects}

The study population was recruited through media advertising. To be qualified as subjects in the study, individuals needed to contact a clinical site within 24 - 36 hours of new onset of cold symptoms, ask to be included in the study, and sign the ICF. Only individuals aged between 18 and 60 years old were eligible. Furthermore, Investigators had to confirm that subjects were generally in good health and that they had at least two different cold symptoms such as runny nose, plugged nose, sneezing, sore throat, scratchy throat, cough, hoarseness, head congestion, chest congestion, or feeling tired. Subjects with medical conditions (e.g. known impairment of the immune function, bleeding disorders, renal diseases, uncontrolled hypertension, angina, and osteoporosis) were excluded. Other exclusion factors included known allergies to Echinacea, Ginger, Goldenrod herb or other plants from the Asteraceae/Compositae/family. Participation in other medical investigations, clinical trials or studies was not allowed. Also excluded from the study were individuals planning a surgery, actively smoking marijuana or with alcohol or drug addiction. Any other condition, which in the opinion of the Investigators, could interfere with the evaluation of the study objectives, or could result in noncompliance with the protocol, was also considered as an exclusion criteria.

Subjects were allowed to use the following medications: acetaminophen (regular Tylenol ${ }^{\circledR}$, but not "Tylenol ${ }^{\circledR}$ Cold", or “Tylenol ${ }^{\circledR}$ Cold and Sinus" or any combination with other products), ibuprofen (Motrin ${ }^{\circledR}$ or $\operatorname{Advil}^{\circledR}$ ), nasal saline spray and multivitamins containing $<100 \mathrm{mg}$ vitamin C. Subjects were asked to discontinue the use and were not allowed to use during the study any experimental therapeutic products, any anticoagulants, diuretics, sedatives or any products intended for the treatment of colds or allergies.

A complete physical examination was performed by the Investigator or his/her designee at Visit 1 (Day 0), Visit 2 (Day 7), and Visit 3 (Day 14) and during unscheduled visits, if applicable. Physical examinations included evaluations of eyes, ears, nose/throat; thorax/ lungs; abdomen; cardiovascular; neurological; musculoskeletal, extremities, skin/dermatology and peripheral vascular. Blood and urine samples were also collected.

\subsection{Randomization Procedures}

A master randomization list was created by using a computer-generated randomization scheme (using the CALL RANUNI1 routine of SAS software (Version 9.2) run by the study statistician). Subjects were randomized in blocks of four and remained in the same cohort for the duration of the study. The randomization list was kept in 
a restricted access location at the Sponsor. Bottles of the Investigation Product (IP) were identified with numbers derived from the master randomization list. Bottles of each IP were supplied to sites in equal proportions.

Bottles were assigned to an eligible subject by choosing the bottle with the lowest number available at the site. The tear-off portion of the bottle's label was attached in the Case Report Form (CRF).

Study personnel were required to fill in box labels with the subject's initials and identification number prior to dispensing the product. The IP was dispensed to subjects by the site's pharmacy or by a member of the study staff.

\subsection{Study Intervention}

BDI-630 is a patent-pending formulation containing a $\mathrm{CO}_{2}$-supercritical extract of Ginger root (Zingiber officinale) and a hydro-alcoholic extract of Goldenrod flower (Solidago virgaurea L.), encapsulated to contain $450 \mathrm{mg} /$ capsule (GOLDaid ${ }^{\mathrm{TM}}$; Biopharmacopae Inc., Canada). The Echinacea control (EC), is a dietary supplement (natural health product) manufactured and commercialized in the US and Canada. The product is available in $250 \mathrm{mg} /$ soft-gel capsules of an extract of Echinacea (Echinacea purpurea), standardized to contain at least two of the following components: cichoric acid, Echinacea alkylamides and Echinacea polysaccharides. The daily softgels were dispensed in bottles of 40 capsules per subject. Bottle labels stated all information required in the study protocol and complied with all applicable regulatory requirements.

All subjects randomized to BDI-630 received the same dose i.e., two capsules (or $900 \mathrm{mg}$ ) twice daily for 10 days. All subjects randomized to EC received the same dose i.e., two capsules (or $500 \mathrm{mg}$ ) twice daily for 10 days. In both cases, the IP was taken with or immediately following a meal. If a dose was missed, subjects were instructed to take it as soon as possible. However, if it was near the time for the next dose, the subject was instructed to skip the missed dose and continue the next scheduled dose. Double doses were not allowed to be taken.

Each subject was randomly allocated in a double-blind fashion to either BDI-630 or EC taken orally as two capsules twice daily with or immediately following a meal, from Day 1 to Day 10.

\subsection{Clinical Outcomes}

The Wisconsin Upper Respiratory Symptom Survey-21 (WURSS-21@2004; University of Wisconsin-Madison Department of Family Medicine. All Rights Reserved by the Wisconsin Alumni Research Foundation.) developed by Barret et al., a 21-item daily questionnaire, was used to evaluate the change in severity of common cold symptoms and quality of life (QoL) [7]. It is composed of two general questions, 10 cold symptoms-related questions (i.e. runny nose, plugged nose, sneezing, sore throat, scratchy throat, cough, hoarseness, head congestion, chest congestion, feeling tired) and 11 health-related questions (i.e. think clearly; sleep well; breathe easily; walk, climb stairs, exercise; accomplish daily activities; work outside the home; work inside the home; interact with others; live one's personal life). Items assessed symptom severity and functional impairment, with a score of 1 considered to be very mild, 3 to be mild, 5 to be moderate and 7 to be severe. Efficacy was assessed by evaluating the severity of the common cold symptoms using the WURSS-21@ questionnaire completed daily by the subject. This questionnaire is widely used, validated, recognized as reliable, accurate and relevant $[7,8]$.

\subsection{Statistical Analysis}

Safety was evaluated on the basis of treatment-emergent adverse events, laboratory measurements (clinical chemistry, hematology, and urinalysis), and concomitant medications.

Efficacy was evaluated by the change in severity of common cold symptoms and the area under the curve (AUC) of the total severity score of the WURSS-21@ for severity of common cold symptoms between Day 1 and 14. The AUC for the WURSS-21@ scores was calculated using a trapezoidal approximation. The change from baseline in total severity score between Day 1 and 14, and the total AUC were compared using two-sample t-tests. WURSS-21@ total severity score was obtained by summing 19 items, excluding the first and last.

\section{Results}

The study protocol as approved aimed to recruit 80 subjects. However, issues related to the H1N1 virus scare, unseasonable cold incidence and the multi-step recruitment method, recruitment was lower than expected. The enrollment period occurred between February to April 2010 and between September 2010 and April 2011 at five sites in Eastern Canada. However, due to issues related to the slow recruitment process after 11 months, the study was halted and the code broken. A total of 47 subjects were screened and 44 subjects were enrolled. The intent-to-treat population (ITT) consisted of 44 patients ( $\mathrm{n}=22$ for BDI-630; $\mathrm{n}=22$ for EC). The modified ITT (mITT) population consisted of 40 patients, excluding 4 patients with major protocol deviations related to study entry inclusion criteria and/or use of prohibited drugs from the efficacy analysis ( $n=3$ for BDI-630 and $n=1$ for EC). The patient overall disposition is presented in Figure 1. 
The majority of patients (over 86\%) were Caucasian with no major racial differences between the 2 groups and the mean age was similar in both groups (35 and 37 years, for BDI-630 and EC, respectively). There was a random imbalance in gender in BDI-630 group (female/ male $=82 \% / 18 \%$ ) while gender was balanced in EC group ( $50 \%$ of each gender). Key demographics are shown in Table 1.

\subsection{Primary Outcome}

The subjects in the BDI-630 group noted worse symptoms at baseline which remained higher throughout the study (Table 2). This difference was confirmed by the WURSS-21(C total AUC (Figure 2/Table 3). The BDI630 group total score was significantly higher when compared to Echinacea. Given that the subjects were randomized into two groups, it is unknown why the BDI630 group noted a greater degree of illness or symptoms before the treatment started.

In order to adjust for baseline differences, the percent change from Day 1 was used in further analysis. The results are plotted in Figure 3. The BDI-630 group showed an appreciable decrease in symptoms by Day 4 which continued throughout the remainder of the study. However, the Echinacea control group did not show any important decrease between Day 1 and Day 7.

Trend analysis for the change scores between Day 1 and Day 7 is represented in Figure 3. This analysis confirms that the decrease in BDI-630 group is much steeper than that for the Echinacea control group (-8.3619 for BDI-630 and -1.1114 for EC). The slope of these two trend lines is significantly different $(\mathrm{p}<0.001)$.

These results were even more pronounced when subjects with major protocol deviations were excluded from analysis (mITT population). As in the ITT population, the WURSS-21@ mean total score at baseline was higher in patients treated with BDI-630 than in patients treated with EC (49.16 and 33.33, respectively Table 2), The WURSS-21@ total score mean \% change from baseline at Day 7 and 14 for BDI-630/EC was $-44 \% / 1 \%$ and $-87 \% /-81 \%$, respectively (Figure 4 ). These changes where especially obvious within the first 7 days. The slope of total score mean \% change from baseline following BDI-630 was 14 times steeper than following EC $(p=0.0002)$, indicating a faster improvement in symptoms following BDI-630 compared to EC during that period of time (Figure 5).

\subsection{Adverse Events}

The subjects tolerated both herbal supplements over the course of the study. During the entire study, 24 adverse events occurred (BDI group $n=14$; EC group $n=10$ ). There was no major intervention related to adverse events reported. Less than $10 \%$ of the BDI group re-

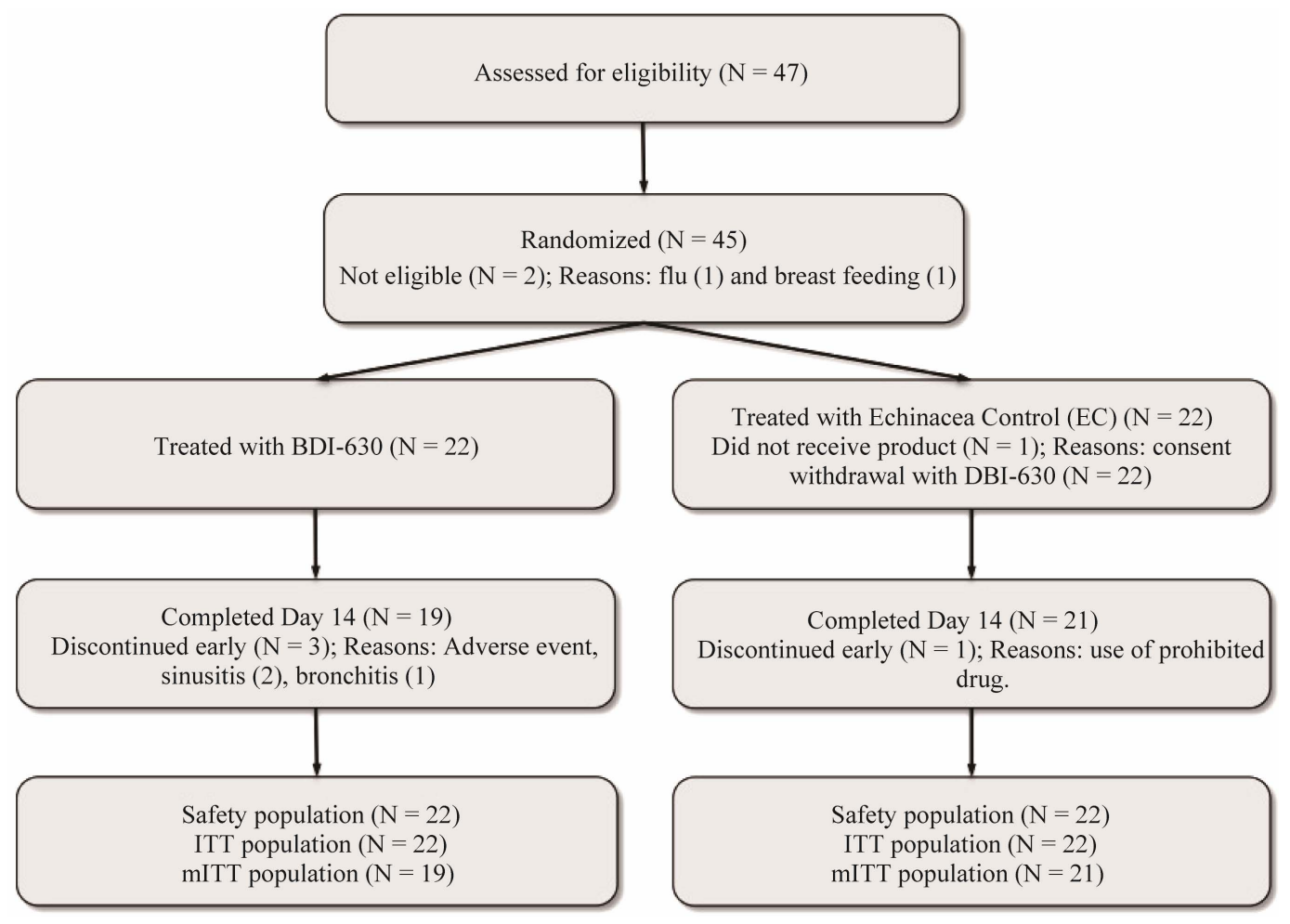

Figure 1. Patient overall disposition. 


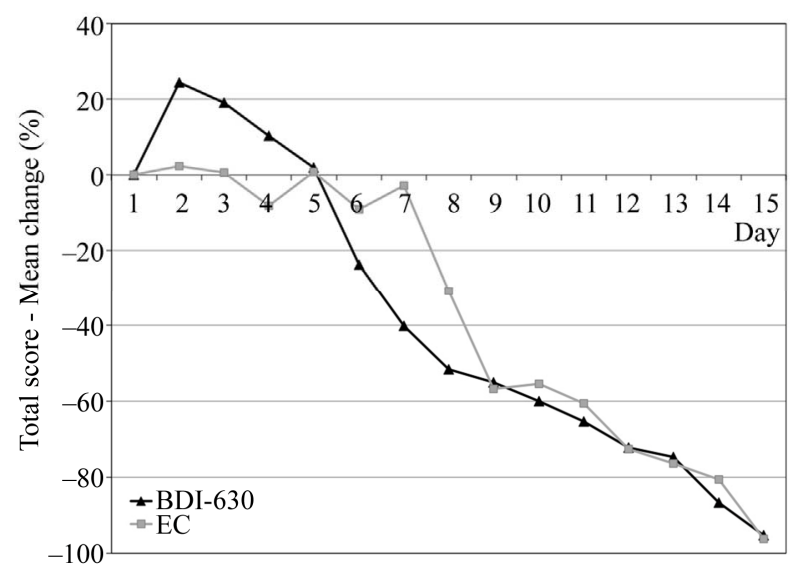

Figure 2. Total score-mean change (\%) from Day 1 (ITT population).

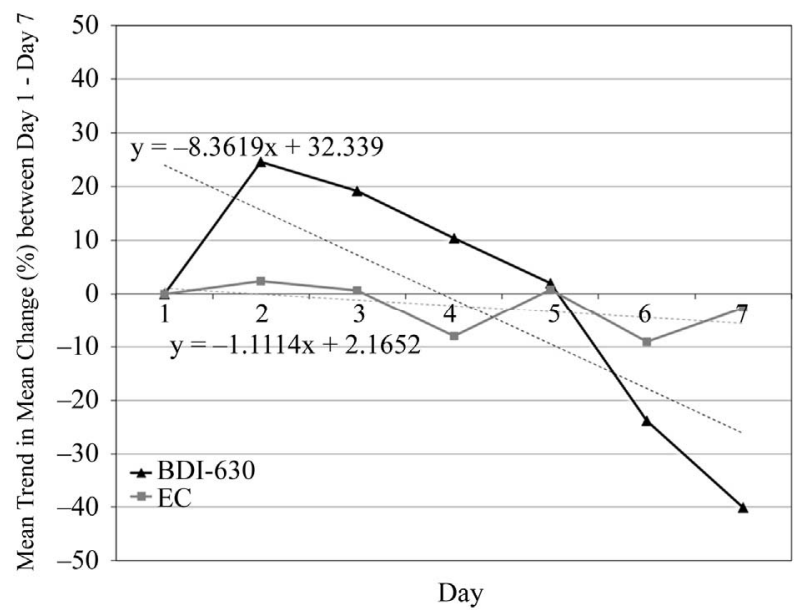

Figure 3. Mean trend in mean changes [\%] between Day 1 and Day 7 (ITT population).

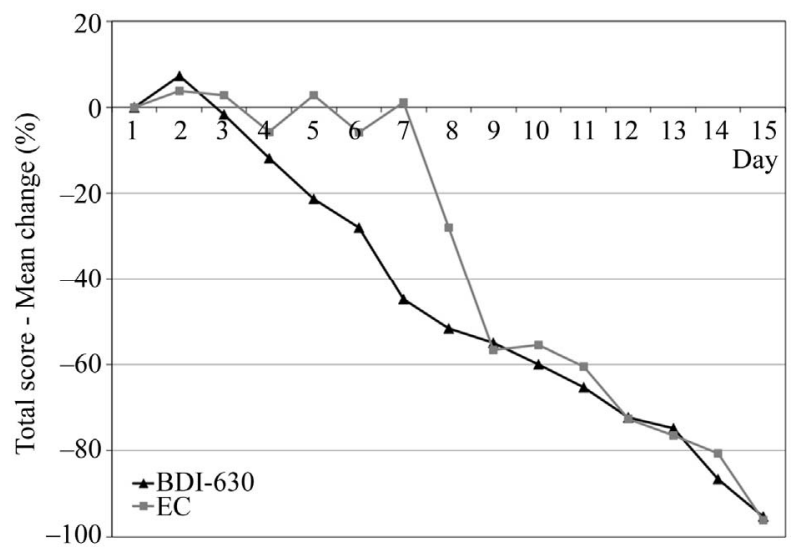

Figure 4. Total score-mean change (\%) from Day 1 (mITT population).

ported mild gastro-intestinal discomfort over the course of the study. The most frequent adverse event following EC was elevated ALT/AST (9\%). None of these symp-

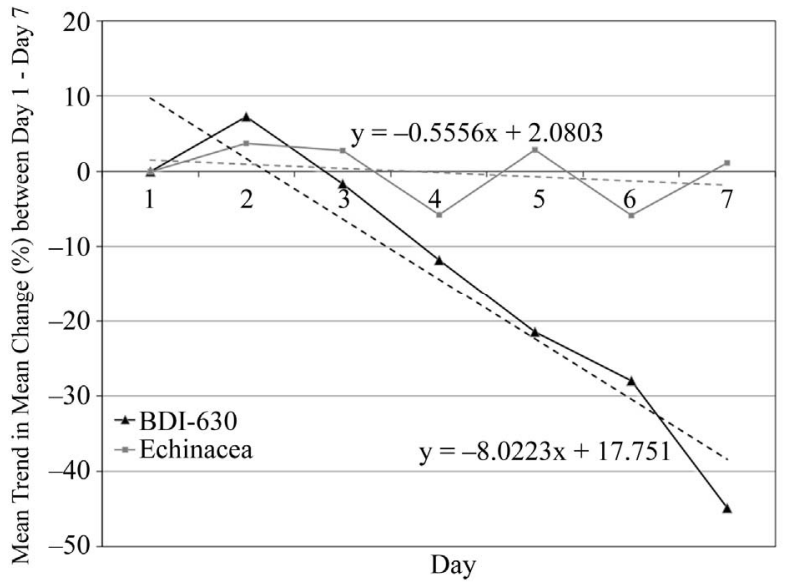

Figure 5. Mean trend in mean changes [\%] between Day 1 and Day 7 (mITT population).

Table 1. Key demographics of the subjects at baseline.

\begin{tabular}{lcc}
\hline & $\begin{array}{c}\text { BDI-630 }(\mathrm{N}=22) \\
\text { (mean } \pm \text { s.d.) }\end{array}$ & $\begin{array}{c}\text { EC }(\mathrm{N}=22) \\
\text { (mean } \pm \text { s.d. })\end{array}$ \\
\hline Age (years) & $35 \pm 9$ & $37 \pm 13$ \\
Sex $(\mathrm{m} / \mathrm{f})$ & $4 / 18$ & $11 / 11$ \\
Height $(\mathrm{cm})$ & $163 \pm 7$ & $167 \pm 6$ \\
Body weight $(\mathrm{kg})$ & $69 \pm 21$ & $75 \pm 18$ \\
BMI $\left(\mathrm{kg} / \mathrm{m}^{2}\right)$ & 24 & 25 \\
\hline
\end{tabular}

s.d. standard deviation.

toms required medical attention, nor did any subject drop for this reason. No serious adverse events were reported during the study.

\section{Discussion}

The results obtained are the first known clinical data for a combination of Ginger and Goldenrod extracts for the treatment of the common cold. Here, individuals taking BDI-630 experienced quicker general cold relief when compared to a natural health product containing a combination of standardized amounts of Echinacea (EC) components. The patent pending extract exhibited a very good safety profile, and the adverse event rate was comparable to that of the EC supplement.

The number of annual reports of common cold and flu, which are both viral in nature, is large, and the economic consequences, considerable; this justifies the need for an effective remedy. Faster alleviation of symptoms and quicker recovery are desirable results. Given that current medical treatments for such conditions are sparse, with the exception of encouraging plenty of fluids and rest, the demand for effective and safe alternatives is warranted.

The current study was under powered and therefore the data must be viewed as exploratory in nature. The recruitment process was compounded by unfortunate tim- 
Table 2. Mean total wisconsin symptoms survey-score (WURSS-21@) at baseline.

\begin{tabular}{|c|c|c|c|c|c|}
\hline & \multicolumn{2}{|c|}{ BDI-630 } & \multicolumn{2}{|c|}{ EC } & \multirow{2}{*}{ p-value* } \\
\hline & $\mathrm{n}$ & mean \pm s.d. & $\mathrm{n}$ & mean \pm s.d. & \\
\hline ITT (baseline) & 22 & $46.6 \pm 18.3$ & 22 & $35.3 \pm 19.9$ & 0.0714 \\
\hline mITT (baseline) & 19 & $49.2 \pm 19.9$ & 21 & $33.3 \pm 18.2$ & 0.0165 \\
\hline
\end{tabular}

${ }^{*}$ t-test; s.d. standard deviation.

Table 3. Total area under the curve (AUC) on the Wisconsin Symptoms Survey (WURSS-21@).

\begin{tabular}{lcccccc}
\hline & \multicolumn{3}{c}{ BDI-630 } & EC & \multicolumn{2}{c}{ p-value* } \\
\cline { 2 - 5 } & $\mathrm{n}$ & mean \pm s.d. & $\mathrm{n}$ & mean \pm s.d. & \\
\hline ITT & 22 & $324.8 \pm 175.3$ & 22 & $194.5 \pm 123.7$ & 0.0071 \\
mITT & 19 & $332.9 \pm 179.5$ & 21 & $190.2 \pm 125.0$ & 0.0069 \\
\hline
\end{tabular}

"t-test; s.d. standard deviation.

ing. The study was conceived and launched during the height of the H1N1 flu global scare. This hindered subject recruitment as did the planned recruitment process within the active clinical sites in Eastern Canada. The study was approved to recruit 80 individuals suffering from cold symptoms, but only 44 patients were enrolled after 11 months. As a consequence, the study was stopped and the code broken.

As shown in Table 3, total area under the curve WURSS-21@ for the mITT population was higher for the BDI-630 group. These subjects presented worse total score at baseline. However, based on the percentage of change of the WURSS-21 C total score from baseline, the BDI-630 group exhibited an impressive and significant pattern of improvement. This was most evident during the initial seven days, and trend analysis between groups showed that the BDI-630 group had a 14-fold steeper slope than EC group. This indicates that the BDI-630 group was recovering faster than the EC group (see Figure 2, Figures 3 and 5).

Recently, Barrett et al. looked the effect of placebo and Echinacea supplementation on the self-reported response to cold symptoms using the total severity questions from WURSS-21@. In a study funded by the National Center for Complementary and Alternative Medicine [9], 719 subjects were randomly assigned into four groups: no intervention, placebo, un-blinded Echinacea, or blinded Echinacea supplements. The subjects received the maximum dose spread out over the first four-days and then followed for another 10 days. The subjects completed the WURSS-21 (C) survey in the morning and evening. The groups did not differ significantly on the total severity index. In addition, the duration until self-reported improvement was approximately 6.34 and 6.76 days in the blinded and un-blinded Echinacea groups which were not statistically different from the corresponding placebo controls, 6.87 and 7.03 days respec- tively. The authors concluded that higher than expected variability limited the power to detect small symptom improvements.

This might be explained by the fact that Echinacea purpurea is thought to function as an immune modulator when warding off the common cold [2,3]. However, immune modulation is not thought to be the most important attribute of the BDI-630 complex. In fact, there is accumulating evidence that subject and its main chemical compounds, sesquiterpenes, zingiberols and gingerols, provide a variety of chemoprotective activities [10]. While the physiological mechanisms of these compounds are not clear, there is a number of studies showing free radical scavenging and anti-oxidant function by subject compounds [10]. Goldenrod has been shown to possess antioxidant activity as well [11]. It seems intuitive that the host response to viral and bacterial agents, inducing cold symptoms, is oxidative in nature. This was highlighted by the pioneering work of Linus Pauling and his work on the role of vitamin $\mathrm{C}$ as an anti-oxidant cofactor in the treatment of common colds [12].

While most common colds are viral in nature, there is evidence of bacterial involvement with some subjects. Kaiser et al. investigated upper respiratory infection in over 300 subjects who presented at outpatient clinics in the UK [13]. The authors concluded that the majority of subjects with upper-respiratory-tract infection do not benefit from antibiotics. However, for individuals whose nasopharyngeal secretions contain $H$. influenzae, $M$. catarrhalis, or $S$. pneumoniae, antibiotics are clinically beneficial. Ginger extracts were reported to possess antibacterial properties that were dose dependent [14]. In addition, a methanol extract of Goldenrod showed antimicrobial activity against S. Aureus, E. Coli and B. cereus [11]. Apáti et al. reported that Goldenrod contained apprecia- ble saponins which were deemed important for its medicinal properties [15]. 
It can be hypothesized that the patent pending Ginger and Goldenrod extract blend in BDI-630 impacts physiological and chemical pathways related to host response and subsequent free radical scavenging. However, in unpublished findings, there is evidence to suggest BDI-630 offers anti-viral protection. BDI-630 decreased plaque formation units (PFU) in Hela cell lines after exposure to Rhinovirus type 1 and type 2. Moreover, BDI630 compared favorably to oseltamivir as a curative compound in CD-1 mice given influenza stain (H1N1) intranasally. The mean time of death for oseltamivir was 7.6 days while that of BDI-630 was 7.8 days and these results were significant compared to other groups in the study (unpublished results). These findings require further discovery and investigation, but the clinical data reported herein suggests that there is some benefit. If a confirmatory study is conducted, the allocation to treatment groups should take into account gender, BMI and a more detailed baseline severity criterion. Assuming that the observed results are representative of the results that would be obtained for a follow-up study, 80 subjects would be sufficient to observe a statistically significant difference at Day 7 in the mean change from Day 1 for the total WURSS $\odot$ score (power $=80 \%$ and alpha error $=5 \%)$.

\section{Conclusion}

In this exploratory study, a patent pending combination of Ginger and Goldenrod extracts (BDI-630) was shown to be superior to Echinacea control (EC) in alleviating cold symptoms particularly within the first seven days of treatment.

\section{Acknowledgements}

The study was funded by Biopharmacopae Design International Inc. (Canada). The authors declare no conflict of interest.

\section{REFERENCES}

[1] A. M. Fendrick, A. S. Monto, B. Nightengale and M. Sarnes, "The Economic Burden of Non-Influenza-Related Viral Respiratory Tract Infection in the United States," Archives of Internal Medicine, Vol. 163, No. 4, 2003, pp. 487-494. doi:10.1001/archinte.163.4.487

[2] P. F. Adams, G. E. Hendershot and M. A. Marano, “Current estimates from the National Health Interview Survey, 1996," Vital and Health Statistics, Vol. 10, No. 200, 1999, pp. 1-212.

[3] R. Nahas and A. Balla, "Complementary and Alternative
Medicine for Prevention and Treatment of the Common cold," Canadian Family Physician, Vol. 57, No. 1, 2011, pp. 31-36.

[4] M. Roxas and J. Jurenka, "Colds and Influenza: A Review of Diagnosis and Conventional, Botanical and Nutritional Considerations," Alternative Medicine Review, Vol. 12, No. 1, 2007, pp. 25-48.

[5] R. Rehman, M. Akram, N. Akhtar, et al., “Zingiber Officinale Roscoe (Pharmacological Activity),” Journal of Medicinal Plants Research, Vol. 5, No. 3, 2011, pp. 344348.

[6] P. Apáti, K. Szentmihályi, S. T. Kristó, et al., "Herbal Remedies of Solidago Correlation of Phytochemical Characteristics and Antioxidative Properties," Journal of Pharmaceutical and Biomedical Analysis, Vol. 32, No. 4-5, 2003, pp. $1045-1053$.

doi:10.1016/S0731-7085(03)00207-3

[7] B. Barrett, R. L. Brown, M. P. Mundt, et al., "Validation of a Short form Wisconsin Upper Respiratory Symptom Survey (WURSS-21)," Health and Quality of Life Outcomes, Vol. 7, No. 1, 2009, p. 76. doi:10.1186/1477-7525-7-76

[8] B. Barrett, R. Brown, M. Mundt, et al., "The Wisconsin Upper Respiratory Symptom Survey Is Responsive, Reliable, and Valid,” Journal of Clinical Epidemiology, Vol. 58, No. 6, 2005, pp. 609-617. doi:10.1016/j.jclinepi.2004.11.019

[9] B. Barrett, R. Brown, D. Rakel, et al., "Echinacea for Treating the Common Cold,” Annals of Internal Medicine, Vol. 153, No. 12, 2010, pp. 769-777.

[10] M. S. Baliga, R. Haniadka, M. M. Pereira, et al., "Update on the Chemopreventative Effects of Subject and Its Phytochemicals," Critical Reviews in Food Science and Nutrition, Vol. 51, No. 6, 2011, pp. 499-523. doi:10.1080/10408391003698669

[11] H. Demir, L. Acik, E. B. Bali, et al., "Antioxidant and Antimicrobial Activities of Solidago Virguaurea Extracts," African Journal of Biotechnology, Vol. 8, No. 2, 2009, pp. 274-279.

[12] L. Pauling, "Vitamin C, the Common Cold and the Flu," Penguin Group, New York, 1981.

[13] L. Kaiser, D. Lew, B. Hirschel, et al., "Effects of Antibiotic Treatment in the Subset of Common-Cold Subjects Who Have Bacteria in Nasopharyngeal Secretions," The Lancet, Vol. 347, No. 9014, 1996, pp. 1507-1510. doi:10.1016/S0140-6736(96)90670-4

[14] S. P. Malu, G. O. Obochi, E. N. Tawo and B. E. Nyong, "Antibacterial Activity and Medicinal Properties of Subject (Zingiber Officinale)," Global Journal of Pure and Applied Sciences, Vol. 15, No. 3, 2009, pp. 365-368.

[15] P. Apáti, P. J. Houghton and A. Kéry, "HPLC Investigation of Antioxidant Components in Solidago Herbal," Acta pharmaceutica Hungarica, Vol. 74, No. 4, 2004, pp. 223-231. 